\title{
Prediction of Shock-Standoff Distance and Entropy Distribution for Forward-Facing Cavity
}

\author{
Gang Wang*, Yanguang Yang, Xiaowei Ma, Tao Jiang, Hongming Gong, Rongzong Kong \\ Hypervelocity Aerodynamics Institute, China Aerodynamics Research and Development Center, Mian Yang, China \\ Email address: \\ wanggang@cardc.cn (Gang Wang), yyyyyggg@sina.com (Yanguang Yang),mxwdyx@gmail.com (Xiaowei Ma), \\ tjdyx@gmail.com (Tao Jiang),64659374@qq.com (Hongming Gong), 13890156659@139.com (Rongzong Kong) \\ ${ }^{*}$ Corresponding author
}

\section{To cite this article:}

Gang Wang, Yanguang Yang, Xiaowei Ma, Tao Jiang, Hongming Gong, Rongzong Kong. Prediction of Shock-Standoff Distance and Entropy Distribution for Forward-Facing Cavity. International Journal of Astrophysics and Space Science. Vol. 6, No. 3, 2018, pp. 52-61.

doi: $10.11648 /$ j.ijass.20180603.11

Received: July 14, 2018; Accepted: July 16, 2018; Published: August 9, 2018

\begin{abstract}
Experiments are performed on a cylinder with a forward-facing cavity at $M_{\infty}=10$ in the FD-14A shock tunnel. The shock-standoff distance and oscillation characteristics are recorded by a high-speed movie, and the dynamic pressure transducer is used to capture the unsteady signal of cavity base. Based on experimental and numerical results, a prediction method for estimating the shock-standoff distance is proposed. Results of shock-standoff distance and oscillation frequency are obtained for experiments in the shock tunnel. The predicted oscillation frequency is in accordance with experimental results. Furthermore, the relation of shock shape and the entropy increase are combined to obtain the characteristics of entropy distribution. As the shock-shape of flat-nosed cylinders is more liable to be influenced than blunt-nosed cylinders with increasing Mach number, the location of the extreme value moves to the surface as the Mach number increases for flat-nosed cylinders, while it remains the identical location for blunt-nosed cylinders.
\end{abstract}

Keywords: Shock-Standoff Distance, Forward-Facing Cavity, Pressure Oscillation, Entropy Distribution

\section{Introduction}

Hypersonic vehicles such as hypervelocity projectiles, re-entry vehicles and hypersonic aircraft are designed to withstand severe heat loads. A proposed heat transfer reduction mechanism is to locate a forward-facing cavity at the nose tip [1-5]. It was reported that the heat flux at the cavity base could be as little as 2 to 10 times less than the stagnation point of a conventional convex hemispherical tip [6]. Hartmann et al. [7] found that placing a forward-facing cavity at a nose tip would produce intense pressure oscillations at a discrete frequency in supersonic flows (the Hartmann whistle). Ladoon et al. [8] conducted an experimental study on a blunt nose with a forward-facing cavity in a Mach 4 quite-flow wind tunnel, and a laser system was employed to perturb the freestream. As the cavity length increases, the resonant frequency decreases. Sambamurthiet et al. [9] investigated the flow field behaviour around a cone with a cavity at hypersonic flows. The results indicated that the frequency of the bow shock is inversely proportional to the cavity depth. Marquart et al. [10] investigated the dynamic of a detached bow shock and the acoustic resonance in a forward-facing nose cavity of a blunt-faced model. They found that the primary mode of oscillation in the cavity is consistent with the classic Organ-pipe frequency. Engblom et al. [11] performed experiments at flow field of Mach 4 and the results showed that the oscillation is spontaneous for deep cavities and mainly affected by the perturbation of the free stream for shallow cavities. The oscillation of high intensity and discrete frequency makes it harder to simulate the flow field and, to some extent, control the vehicle.

However, most of the researches aimed at the issue of heat flux reduction and unsteady phenomena in cavities. Only a few papers had focused on shock behaviours for forward-facing cavities. Huebner et al. [6], Sambamurthi et al. [9], Marquart et al. [10] and Engblom et al. [12] measured 
shock-standoff distance using optical methods. As the shock-standoff distance varies for different dimensions of cavities, it is fundamental to predict the standoff distance for estimating the oscillation frequency. Yuceil et al. [13] applied the prediction method for blunt-nosed cone without cavity to approximate one with a cavity, while this may cause inaccuracy to some extent. The main objectives of the present study are to 1) develop a prediction method for various dimensions of forward-facing cavities, 2) study the characteristics of entropy distribution. The details of this study are discussed in the subsequent sections.

\section{Experimental Apparatus}

\subsection{Test Facility}

The experiments were conducted in FD-14A shock tunnel (Figure 1) in CARDC (China Aerodynamics Research and Development Center). The FD-14A shock tunnel produces flows from Mach 6 to 16 with its reservoir pressure up to $69 \mathrm{Mpa}$ and the total temperature up to $4000 \mathrm{~K}$. The available test time is about $15 \mathrm{~ms}$. Typical test conditions of the experiment are given in Table 1. The experiments were conducted at a freestream Mach number of 10 and the unit Reynolds number $R e_{\infty \mathrm{L}}$ of $7.4 \times 10^{6} / \mathrm{m}$.

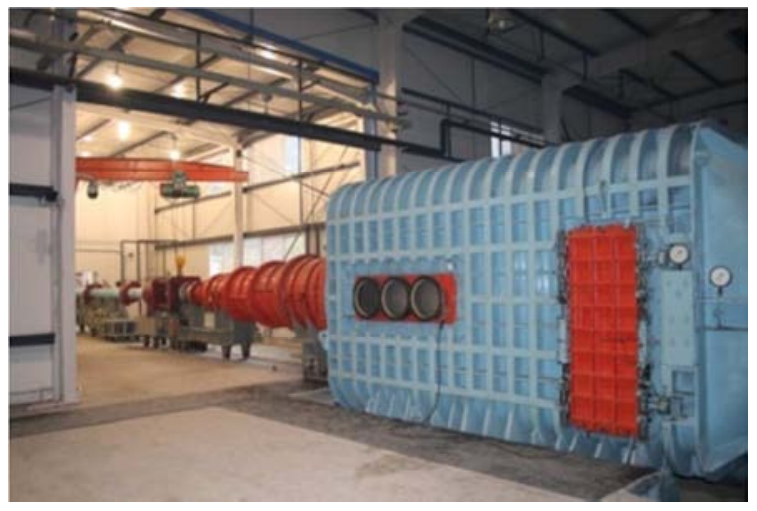

Figure 1. FD-14A shock tunnel in CARDC.

Table 1. Test conditions in the FD-14A shock tunnel.

\begin{tabular}{llll}
\hline $\boldsymbol{M}_{\infty}$ & $\boldsymbol{P}_{\mathbf{0}}(\mathbf{M p a})$ & $\boldsymbol{T}_{\mathbf{0}}(\mathbf{K})$ & $\boldsymbol{R} \boldsymbol{e}_{\infty \mathbf{L}} /(\mathbf{m})$ \\
\hline 6 & $2 \sim 12$ & 800 & $8.1 \times 10^{6} \sim 6.7 \times 10^{7}$ \\
9 & $3 \sim 69$ & $800 \sim 3200$ & $1.0 \times 10^{6} \sim 5.5 \times 10^{7}$ \\
10 & $3 \sim 69$ & $900 \sim 4000$ & $7.5 \times 10^{5} \sim 4.5 \times 10^{7}$ \\
12 & $3 \sim 50$ & $1200 \sim 4000$ & $4.0 \times 10^{5} \sim 9.0 \times 10^{6}$ \\
14 & $5 \sim 40$ & $1600 \sim 4000$ & $2.0 \times 10^{5} \sim 4.0 \times 10^{6}$ \\
16 & $5 \sim 40$ & $1900 \sim 4000$ & $1.5 \times 10^{5} \sim 1.0 \times 10^{6}$ \\
\hline
\end{tabular}

\subsection{Test Model and Techniques}

A flat-nosed cylinder, $300 \mathrm{~mm}$ in diameter, with a cavity at the tip is shown in Figure 2 (Schematic is given instead of photos of the model for the reason of the project). The cavity diameter $D$ is $80 \mathrm{~mm}$ and its depth $L$ is $200 \mathrm{~mm}$. A dynamic pressure transducer (NS-2) with the frequency response of $300 \mathrm{KHz}$ and the range of $0-50 \mathrm{Kpa}$ were used. The transducer is nominally $2 \mathrm{~mm}$ in diameter and is installed with its axes parallel to the model axis, and flush mounted with the base of the cavity. The signals were amplified by DH3840Q Amplifiers and pass through DH5862A Electronic Filters with a cutoff frequency set at $300 \mathrm{KHz}$. A high-speed camera is used to record the shock wave at the rate up to $5000 \mathrm{frames} / \mathrm{s}$. The camera is with largest pixels of $1024 \times 1024$ and largest frame frequency. A motion analyzer was used to measure the time-dependent shock-standoff distance.

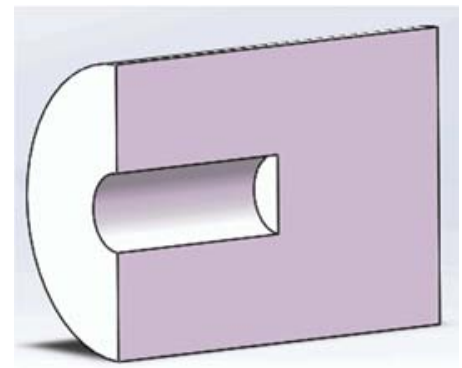

Figure 2. Schematic drawing of the forward-facing cavity in the FD-14A shock tunnel.

\section{Methodology}

\subsection{Shock-Standoff Distance}

Based on experimental results, Billig [14] established the correlations for blunt-nosed cones and flat-nosed cylinders and assume a hyperbolic shock shape given by

$$
\bar{x}=1+\bar{s}-\overline{R_{\mathrm{c}}} \cot ^{2} \beta[H-1]
$$

In Eq. (1), $H=\left(1+\frac{\bar{y}^{2} \tan ^{2} \beta}{{\overline{R_{\mathrm{c}}}}^{2}}\right)^{0.5}$. The nomenclature in Eq. (1) is illustrated in Figure 3. $\overline{R_{\mathrm{c}}}$ is the radius of the curvature of the shock wave at the vertex of the hyperbola; $\bar{s}$ is the shock-standoff distance; $\bar{x}$ and $\bar{y}$ are Cartesian coordinate. These parameters are all nondimensionalized by the radius of the nose $R . \beta$ is the wave angle of the shock wave in the limit of an infinite distance away from the nose. If the downstream body is a cylinder, then $\beta$ is the angle of Mach wave.

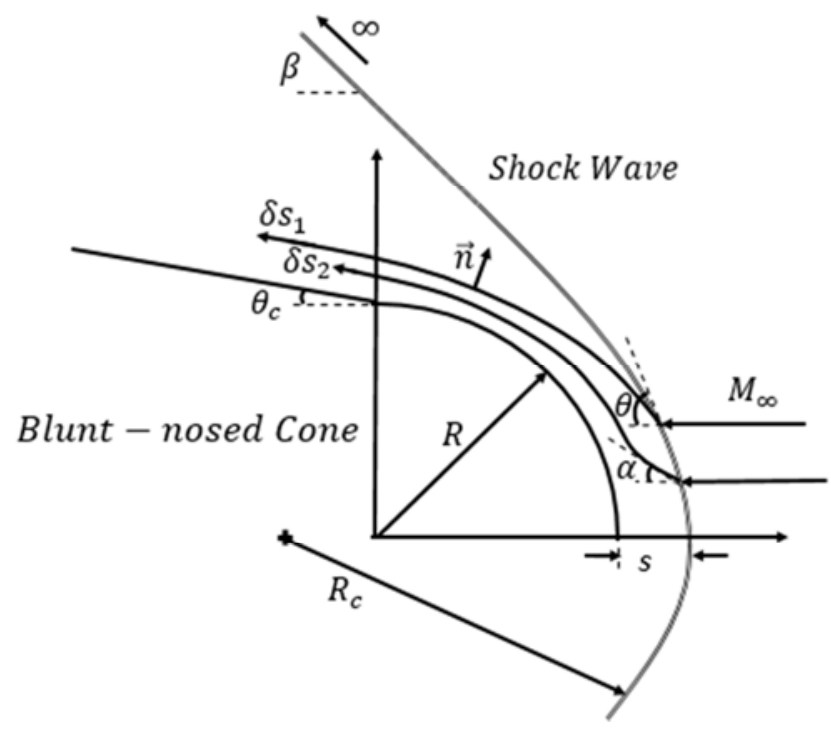




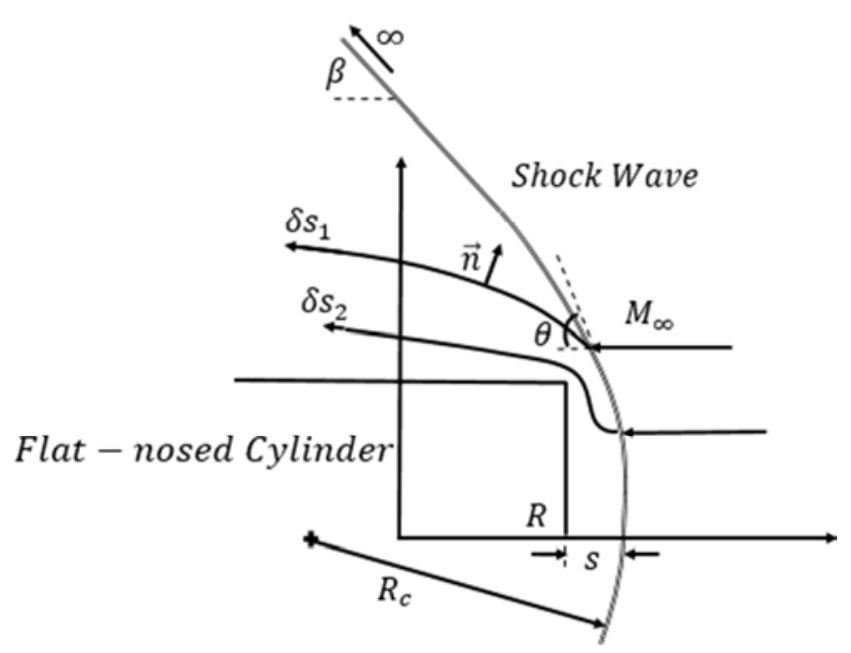

Figure 3. Nomenclature for the shock wave of blunt-nosed cones (up) and flat-nosed cylinders (down).

In Eq. (1), $\bar{s}$ and $\overline{R_{\mathrm{c}}}$ are correlated from experimental results as

$$
\begin{gathered}
\text { Blunt cones: } \bar{s}=0.143 \mathrm{e}^{3.24 / M_{\infty}^{2}} \\
\text { Flat cylinders: } \bar{s}=0.386 \times \mathrm{e}^{4.67 / M_{\infty}^{2}} \\
\text { Blunt cones: } \overline{R_{\mathrm{c}}}=1.143 \mathrm{e}^{0.54 /\left(M_{\infty}-1\right)^{1.2}} \\
\text { Flat cylinders: } \overline{R_{\mathrm{c}}}=1.386 \times \mathrm{e}^{1.8 /\left(M_{\infty}-1\right)^{0.75}}
\end{gathered}
$$

It is obvious that the shock-standoff distance $s$ mainly depend on two factors: Mach number and geometry (radius of the nose).

However, under the condition of varied cavity diameter $D$ and nose diameter $D_{\mathrm{n}}$, the shock-standoff distance may change because the distance is influenced by geometry. For blunt-nosed cones, 1) under the condition of relatively small $D / D_{n}$, the geometric characteristics behave similarly with the one without cavity, hence the shock-standoff distance is almost equal to the one without a cavity. 2) As $D / D_{n}$ increases to a medium value, the central part of detached shock is nearly parallel to the base of the cavity, while the other part of the shock remains the same shape of the blunt-nosed cone without a cavity. 3) For relatively large $D / D_{n}$, Sambamurthi et al. [9] argued the detached shock is almost parallel to the base of the cavity, and what is more, the shape of the whole shock is somewhat similar to flat-nosed cylinder's shock wave. Based on these phenomena and Eq. (1) (3), three equations for predicting shock-standoff distance are proposed for three different cases:

For case 1, as the shock shape is quite similar to blunt-nosed cone without a cavity, the shock-standoff distance is given by

$$
s /\left(\frac{1}{2} D_{n}\right)=0.143 \exp \left(3.24 / M_{\infty}{ }^{2}\right)
$$

For case 2, as the central part of shock is nearly parallel to the base of the cavity, and hence the shock shape is similar to the flat-nosed cylinder whose radius equals to $\frac{1}{2} D$. Therefore, the shock-standoff distance is approximated by

$$
s /\left(\frac{1}{2} D\right)=0.386 \exp \left(4.67 / M_{\infty}{ }^{2}\right)
$$

For case 3 , it is somewhat different from case 2 . The most part of the shock for relatively large cavity behaves like a flat-nosed cylinder. However, the radius of the flat-nosed cylinders approximated by the definition of an equivalent radius of $\frac{\sqrt{2}}{4} D_{n}$, and accordingly the shock-standoff distance for case 3 is

$$
s /\left(\frac{\sqrt{2}}{4} D_{n}\right)=0.386 \exp \left(4.67 / M_{\infty}^{2}\right)
$$

\subsection{Oscillation Frequency}

Experimental and numerical investigations have consistently found that cavity configurations exhibit oscillatory flow due to resonance inside the cavity. The primary resonance frequency can be calculated from simple linear theory given the cavity depth and the speed of sound inside the cavity. In classic Organ-pipe Theory, the wavelength $\lambda$ of the primary resonance is given as $\lambda=4 L$, where $L$ is the cavity depth. Thus the frequency $f$ corresponding to the wavelength can be obtained knowing the speed of sound $a$. Assuming that the gas temperature inside the cavity is approximately the total temperature $T_{0}$ of the flow, which leads to $a=\sqrt{\gamma R_{\text {con }} T_{0}}$. Hence

$$
f=\sqrt{\gamma R_{\text {con }} T_{0}} / 4 L
$$

where $\gamma$ is the ratio of specific heats and $R_{\text {con }}$ is the specific gas constant [10].

However, some researchers [11, 13, 15-17] suppose that the sound wave is oscillating between the base of the cavity and average position of the shock wave, Hence the $L$ in Eq. (9) should be corrected to $L^{*}=L+S$. This issue would be discussed later.

\subsection{Entropy Distribution}

For a certain model, the shock shape is in steady state for a given free-stream Mach number. From Eq. (1), the relation of the local shock-wave angle $\theta$ and the location of the shock wave is

$$
\frac{\mathrm{d} \bar{y}}{\mathrm{~d} \bar{x}}=-\tan \theta=-\left[\left(\frac{\overline{R_{\mathrm{c}}}}{\bar{y}}\right)^{2}+\tan ^{2} \beta\right]^{0.5}
$$

For $\bar{y} \rightarrow \infty, \theta \rightarrow \beta$. Considering the upper half plane for convenience as the flow is symmetric to the $x$ coordinate, the shock wave could be regarded as countless parts of oblique shock waves (nearly normal shock wave around stagnation region). For the flow through an oblique shock wave, $T$ is the variable of static temperature, $p$ is the variable of static pressure. The upstream condition is denoted by subscript $\infty$ and the downstream condition is denoted purely by parameter variable itself

$$
\begin{gathered}
\frac{T}{T_{\infty}}=\frac{\left(2 \gamma M_{\infty}^{2} \sin ^{2} \theta-\gamma+1\right)}{\gamma+1} \frac{\left[(\gamma-1) M_{\infty}^{2} \sin ^{2} \theta+2\right]}{(\gamma+1) M_{\infty}^{2} \sin ^{2} \theta} \\
\frac{p}{p_{\infty}}=1+\frac{2 \gamma}{\gamma+1}\left(M_{\infty}^{2} \sin ^{2} \theta-1\right)
\end{gathered}
$$

In inviscid iso-energetic flow, the entropy increases across 
the shock wave. Downstream the shock wave, the entropy could be considered as constant along the streamlines. The entropy increase across the shock wave would be different from one streamline to the next. For a given free-stream Mach number, the normal part leads to the largest entropy increase, while the entropy increase is smaller across the oblique-shock part and actually becomes smaller and smaller with decreasing shock angle until the shock angle approaches the Mach angle. The entropy increase across the shock wave $\delta S$ is

$$
\delta S=\frac{\mathrm{d} h}{T}-\mathrm{R}_{\operatorname{con}} \frac{\mathrm{d} p}{p}
$$

In Eq. (13), $h$ is the enthalpy of air.

From Eq. (11) and Eq. (12), we have $\mathrm{d} T$ and $\mathrm{d} p$, respectively

$$
\begin{gathered}
\frac{\mathrm{d} T}{T}=\frac{4(\gamma-1)\left(\gamma M_{\infty}^{4} \sin ^{4} \theta+1\right) d \theta}{\tan \theta\left(2 \gamma M_{\infty}^{2} \sin ^{2} \theta-\gamma+1\right)\left[(\gamma-1) M_{\infty}^{2} \sin ^{2} \theta+2\right]} \\
\frac{\mathrm{d} p}{p}=\frac{4 \gamma M_{\infty}^{2} \sin \theta \cos \theta}{2 \gamma M_{\infty}^{2} \sin ^{2} \theta-\gamma+1} d \theta
\end{gathered}
$$

$\mathrm{d} \theta$ could be obtained from Eq. (10) as

$$
\mathrm{d} \theta=-\frac{\overline{R_{C}^{2}} \cos ^{3} \theta}{\bar{y}^{3} \sin \theta} \mathrm{d} \bar{y}
$$

Substituting Eq. (14), Eq. (15) and Eq. (16) into Eq. (11), we obtain

$$
\frac{\mathrm{d} S}{\mathrm{~d} \bar{y}}=f\left(M_{\infty}, \overline{R_{\mathrm{c}}}, \bar{y}, \beta\right)
$$

The Eq. (17) is the entropy gradient as the streamlines just flow across the shock wave. As the expression of $\mathrm{d} S / \mathrm{d} \bar{y}$ is somewhat complicated which may occupy the space, but it is not difficult to illustrate the expression. In particular, for a calorically perfect gas within shock layer, the specific heats at constant pressure $c_{\mathrm{p}}$ is constant, Eq. (17) could be written as

$$
\frac{\mathrm{d} S}{\mathrm{~d} \bar{y}}=\frac{-B\left(M_{\infty}^{2} \sin ^{2} \theta-1\right)^{2}}{\left(2 \gamma M_{\infty}^{2} \sin ^{2} \theta-\gamma+1\right)\left[(\gamma-1) M_{\infty}^{2} \sin ^{2} \theta+2\right]}
$$

In Eq. (18), $B=\frac{4 \gamma{\overline{R_{\mathrm{c}}}}^{2} R_{\operatorname{con}} \cos ^{4} \theta}{\sin ^{2} \theta}$

Integrating Eq. (13), we obtain the entropy distribution behind the shock wave

$$
\delta S=S-S_{\infty}=\int c_{\mathrm{p}} \frac{\mathrm{d} T}{T}-R_{\text {con }} \ln \frac{p}{p_{\infty}}
$$

$S$ represents the entropy at a given temperature $T$ and pressure $p$, and $S_{\infty}$ represents the entropy of incoming flow.

The entropy layer introduces a large amount of rotationality into inviscid flows over bodies, as can be quantitatively obtained from Crocco's theorem, which could be written as follows

$$
\frac{\partial \vec{u}}{\partial t}-T \nabla S+\vec{w} \times \vec{u}+\nabla h_{0}=0
$$

In Eq. (20), $\vec{u}$ is the local velocity vector along the streamline; $h_{0}$ is the total enthalpy. For steady and adiabatic flows considered here, $h_{0}$ is constant, and hence $\nabla h_{0}=0$. The vorticity $\vec{w}$ is related to the entropy gradient $\nabla S$ normal to a streamline

$$
T \nabla S=\vec{w} \times \vec{u}
$$

In Eq. (21), $\nabla S=\frac{\mathrm{d} S}{\mathrm{~d} n} \vec{n}$, in which $\vec{n}$ is perpendicular to the local surface, hence

$$
\frac{\mathrm{d} S}{\mathrm{~d} n}=\frac{1}{\cos \alpha} \frac{\mathrm{d} S}{\mathrm{~d} \bar{y}}
$$

In Eq. (22), $\alpha$ is the turning angle as streamlines flow through the shock wave. Relation of $\alpha$ and $\theta$ is

$$
\tan \alpha=2 \operatorname{ctan} \theta \frac{M_{\infty}^{2} \sin ^{2} \theta-1}{M_{\infty}^{2}(\gamma+\cos (2 \theta))+2}
$$

Referring to Figure 3, considering the case of a plane flow (the arguments could be easily extended to axisymmetric flows), the tangential component $u_{\tau}$ and the normal component $u_{n}$ of velocity along the streamlines are

$$
\begin{gathered}
u_{\tau}=u_{\infty} \cos \theta \\
u_{n}=u_{\infty} \sin \theta \frac{\left.(\gamma-1) M_{\infty}^{2} \sin ^{2} \theta+2\right)}{(\gamma+1) M_{\infty}^{2} \sin ^{2} \theta}
\end{gathered}
$$

Combining the Eq. (17), Eq. (21) and Eq. (22), the vorticity distribution as the streamlines just flow across the shock wave is

$$
\vec{w}=\frac{T}{u} \frac{\partial S}{\partial n} \frac{\vec{u} \times \vec{n}}{|\vec{u} \times \vec{n}|}
$$

\section{Results and Discussion}

\subsection{Shock-Standoff Distance}

Now the Eq. (6) Eq. (8) would be validated for forward-facing cavities by experimental results.

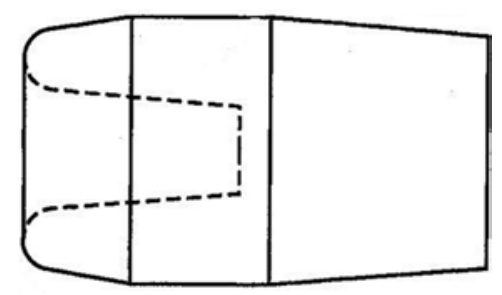

Figure 4. Schematic drawing of a forward-facing Cavity [10].

Huebner et al. [6], Sambamurthi et al. [9] and Marquart et al. [10] conducted experiments at Mach number of 10 on the models who have similar geometry but differ in scales (Figure 4). The parameter of $D / D_{\mathrm{n}}$ equals 0.67 . According to the varied depth of the cavity, the model could be divided into "deep cavity", "medium cavity" and "shallow cavity". In Figure 5, it is shown the shock-standoff distance varies as the function of cavity depth. "E1", "E2", and "E3" in the figure denotes the prediction results for Case 1,2, and 3, respectively, hereinafter the same. Now that the depth varies for $L=62 \mathrm{~mm}$ and $L=125 \mathrm{~mm}$, the standoff distances almost remain the same for two cases as they are characterized by the same geometry of blunt-nosed cone. For $L=187.5 \mathrm{~mm}$, the model is larger than the other two cases, hence the shock-standoff distance is larger than the other two cases. In general, forecasting results indicate that "E3" predicts shock-standoff distance accurately 
for a forward-facing cavity with varied geometry and varied cavity depth for $D / D_{n}=0.67$, while results of "E1" and "E2" underestimate the shock-standoff distance.

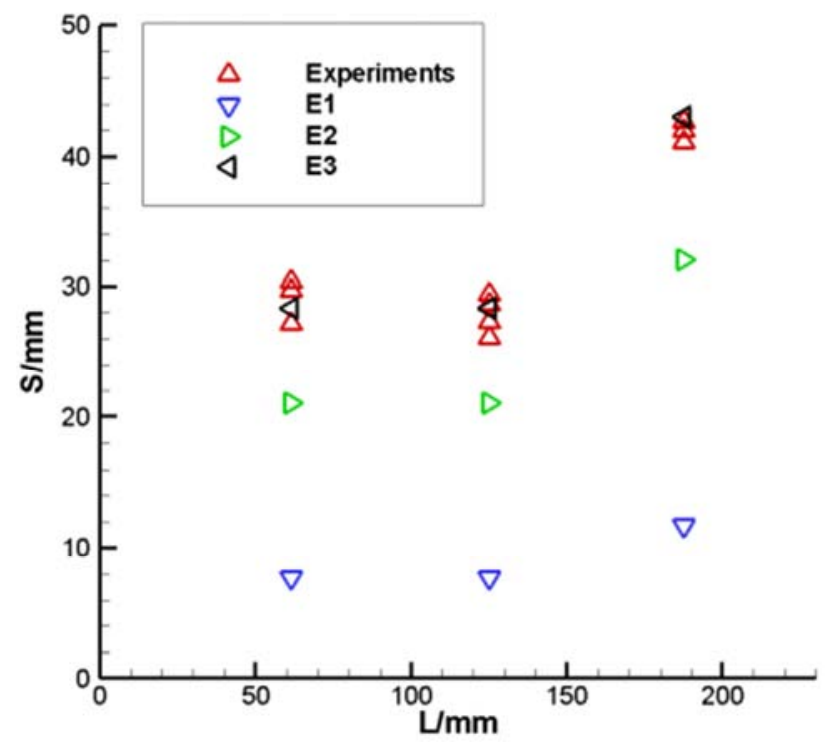

Figure 5. Comparison of the predicted shock-standoff distance and experimental results $\left(D / D_{n}=0.67\right)$.

Engblom et al. [12] and Saravanan et al. [18] performed experiments at Mach number of 5 and 8 on three models of $D / D_{\mathrm{n}}=0.17, D / D_{\mathrm{n}}=0.28$ and $D / D_{\mathrm{n}}=0.5$. As shown in Figure 6 , for $D / D_{n}=0.17$, "E1" predicts the experimental result accurately, while "E2" and "E3" fail to predict the standoff distance. For $D / D_{n}=0.28$, "E1" and "E2" are able to predict shock-standoff distance, while the result of "E3" is somewhat larger than experimental results. For $D / D_{n}=0.5$, although the result of "E3" is a little larger than the experimental result, it is more accurate than the other two methods.

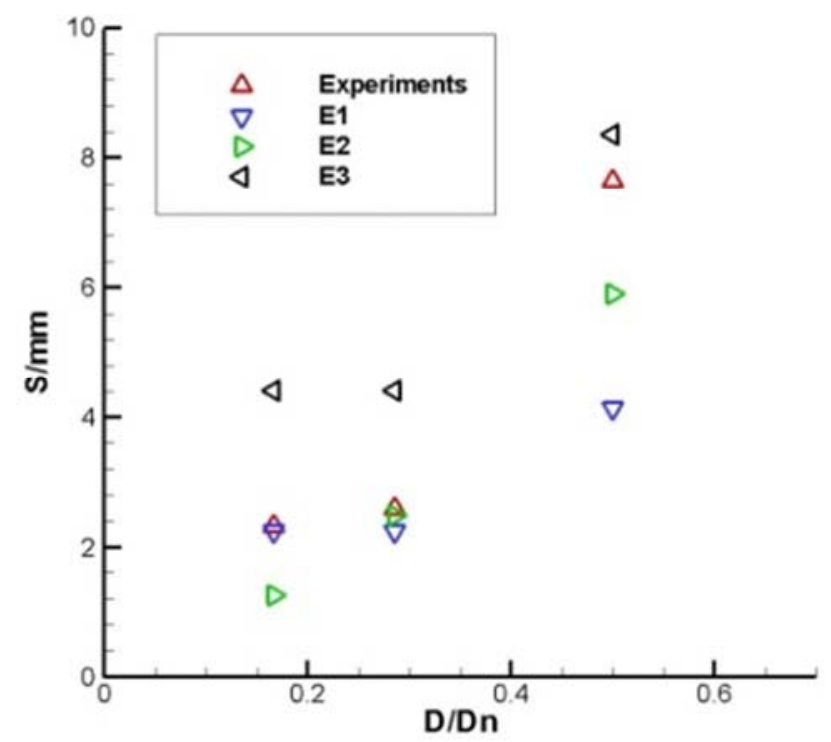

Figure 6. Comparison of the predicted shock-standoff distance and experimental results $\left(D / D_{n}=0.17 \sim 0.5\right)$.

In general, for the case of relatively small $D / D_{n}$, Eq. (6) obtain better results as the existence of cavity weakly affects the shock shape and the shock-standoff distance. For the medium value of $D / D_{n}$, the prediction methods of "E1" and "E2" may be both suitable for predicting the standoff distance. For relatively large $D / D_{n}$, the method "E3" behaves better than the other two methods.

For the flat-nosed cylinder, the shadow photos in FD-14A shock tunnel is shown in Figure 7. The average value of the shock-standoff distance is approximate $88 \mathrm{~mm}$. Applied with equation (3) for the flat-nosed cylinder, the results show that the shock-standoff distance is $63 \mathrm{~mm}$, which deviates from experimental results for about $28 \%$.

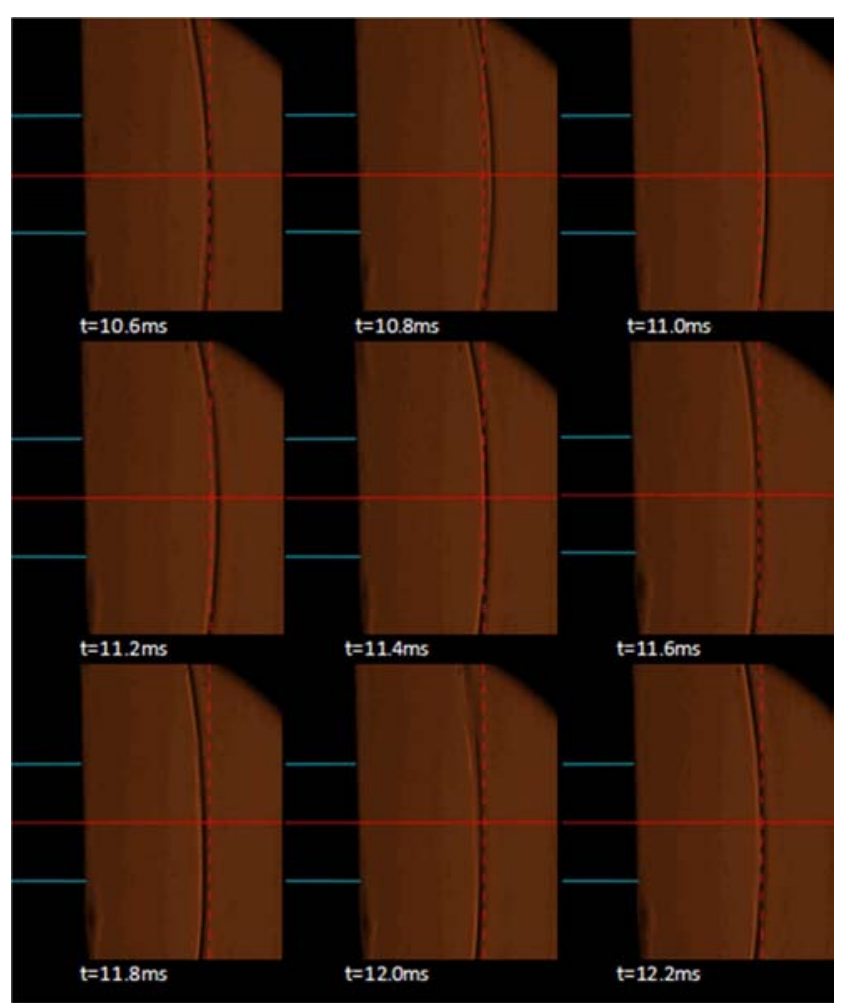

Figure 7. Shock position in FD-14A shock tunnel (one period).

\subsection{Oscillation Frequency}

Controversy exists on the issue of how to define $L$ in the equation $f=a / 4 L$ for estimating the oscillation frequency. The $L$ may be defined as the depth of the cavity or the sum of the cavity depth and shock-standoff distance. Based on experimental and numerical results, the oscillation frequency is predicted using $L$ and $L^{*}$. For the condition of $L^{*}$, the standoff distance is calculated by "E1","E2" and "E3", respectively.

Engblom et al. [12] performed numerical simulations at a Mach number from 3 to 9 . " $L$ " in Figure 8 denotes the prediction results by defining $L$ as the depth of the cavity. For the case of $L \in(2,10) \mathrm{mm}, L / D=0.75$, and $D / D_{n} \in$ $(0.06,0.25)$, the simulation results are just between the result of "E1" and "E2". For the case of $L>12.7 \mathrm{~mm}, L / D \geq 1.4$ and $D / D_{n}=0.5$, the influence of shock-standoff distance on oscillation frequency decreases as the cavity depth increases, while "E3" provides more accurate results of shock-standoff 
distance than two other methods, and hence more accurate results of the oscillation frequency.

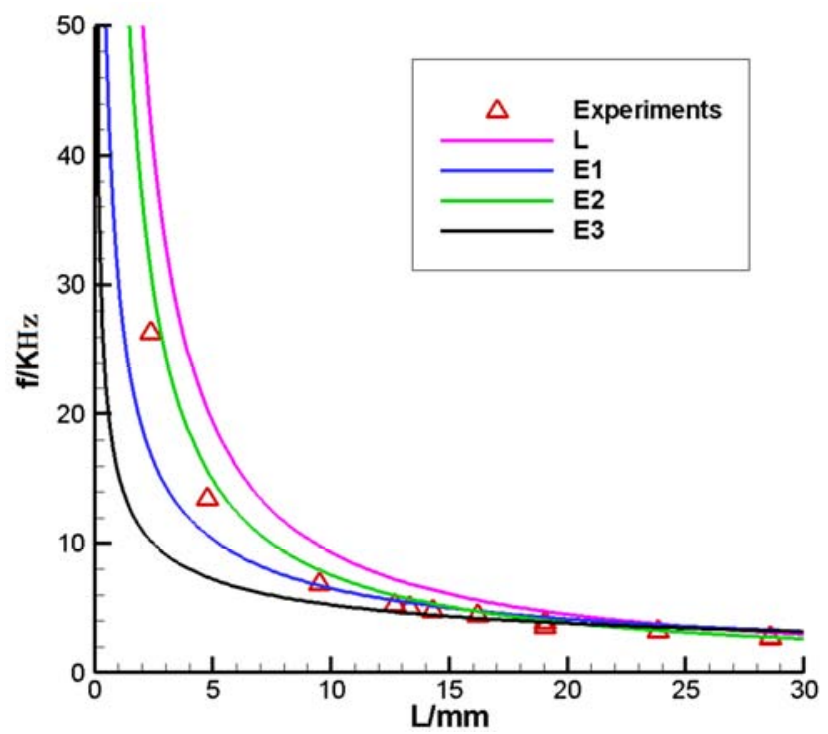

Figure 8. Comparison of the predicted oscillation frequency and simulation results $\left(D / D_{n}=0.06 \sim 0.5\right)$.

Juliano et al. [19] conducted experiments on a forward-facing cavity of $D / D_{\mathrm{n}}=0.5$. The experimental results perfectly agree with the predicted results of "E3", while results for " $L "$, "E1" and "E2" are larger than experimental results (Figure 9).

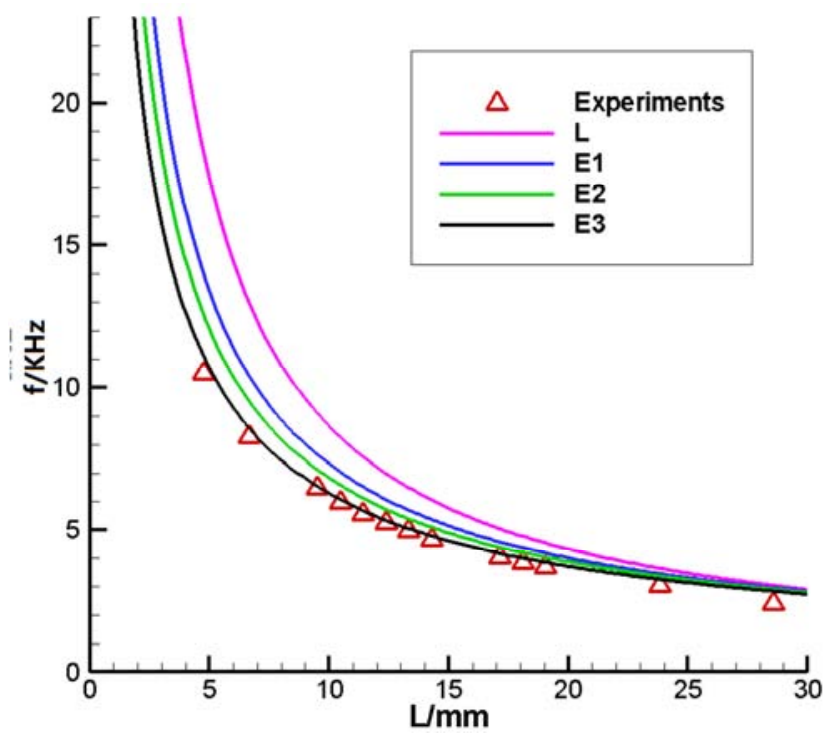

Figure 9. Comparison of the predicted oscillation frequency and experimental results $\left(D / D_{n}=0.5\right)$.

Engblom et al. [16] and Yuceil et al. [17] conducted experiments and numerical simulation. In the case of $L / D \in(0.2,1.2)$ and $D / D_{n}=0.5$, as the cavity depth varies, "E3" predicts the oscillation frequency accurately (Figure 10). For $L / D \in(0.3,1.3)$ and $D / D_{\mathrm{n}}=0.25-0.5$, the result is shown in Figure 11. The range of $L<7 \mathrm{~mm}$ corresponds to the models of $D / D_{\mathrm{n}}=0.25$, and in this case the predicted results by "E3" close to but underestimate the experimental results. For $L>7 \mathrm{~mm}$ and $D / D_{n}=0.5$, the "E3" method predicts the experimental results accurately.

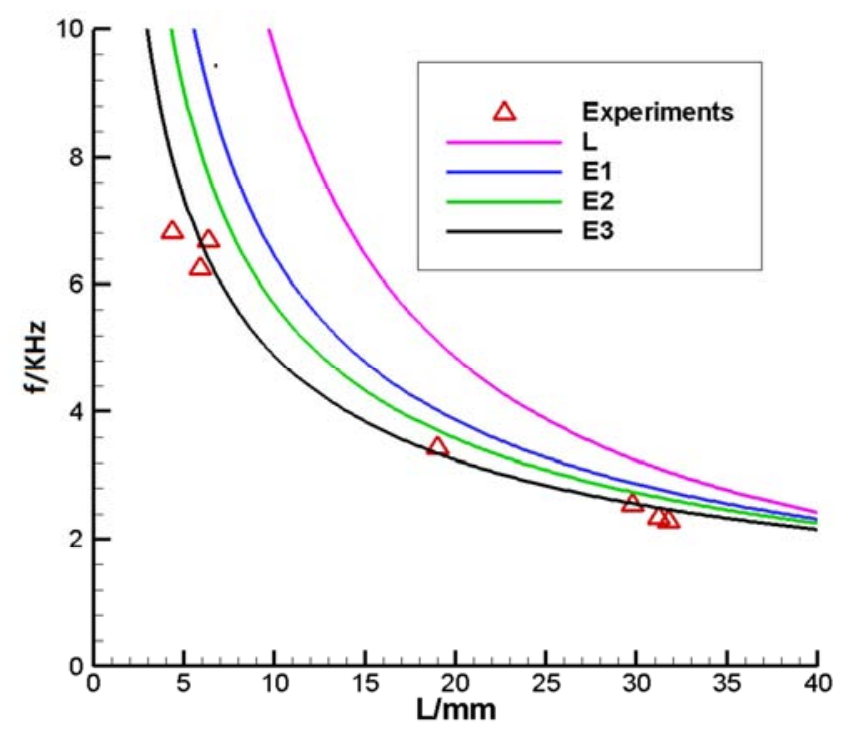

Figure 10. Comparison of the predicted oscillation frequency and experimental results $\left(D / D_{n}=0.5\right)$.

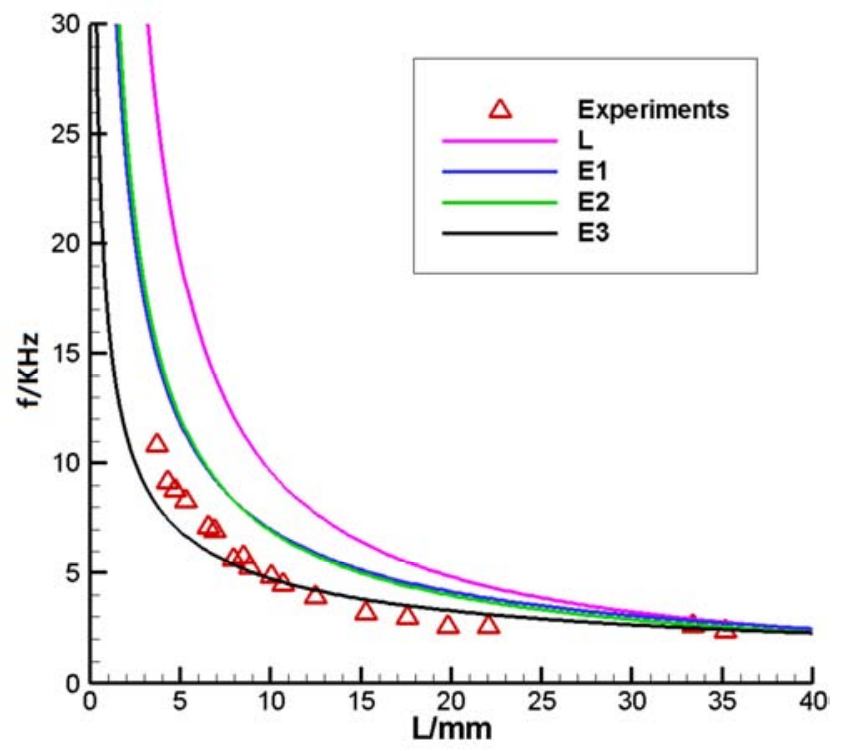

Figure 11. Comparison of the predicted oscillation frequency and experimental results $\left(D / D_{n}=0.25 \sim 0.5\right)$.

Ladoon et al. [8] and Segura [20] performed experiments on a forward-facing cavity of $D / D_{n}=0.5$ and $L / D \in$ $(0.5,3.0)$. The experimental results equal to the results predicted by "E3" method for most of the conditions (Figure 12).

Figure 13 shows pressure signals measured at the base of the cavity in FD-14A. The pressure signals have been normalized by the total pressure downstream of the shock wave. The oscillation frequency is approximate $622 \mathrm{~Hz}$. According to Organ-pipe Theory, the oscillation frequency can be calculated to be $560 \mathrm{~Hz}$. Furthermore, the result based on Eq. (8) for the flat-nosed cylinder is about $614 \mathrm{~Hz}$. It is obvious that predicted result of is close to the results of 
pressure measurement.

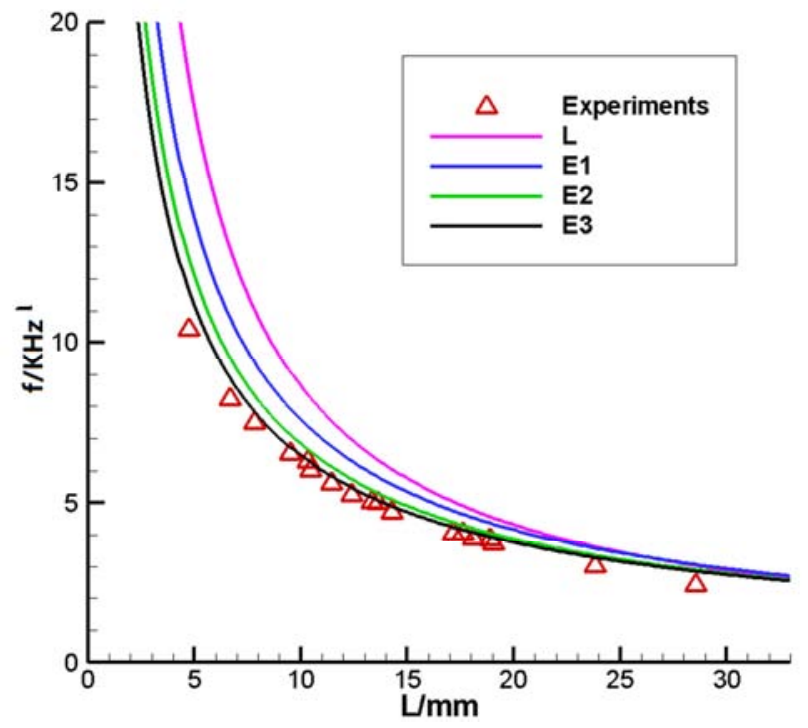

Figure 12. Comparison of the predicted oscillation frequency and experimental results $\left(D / D_{n}=0.5\right)$.

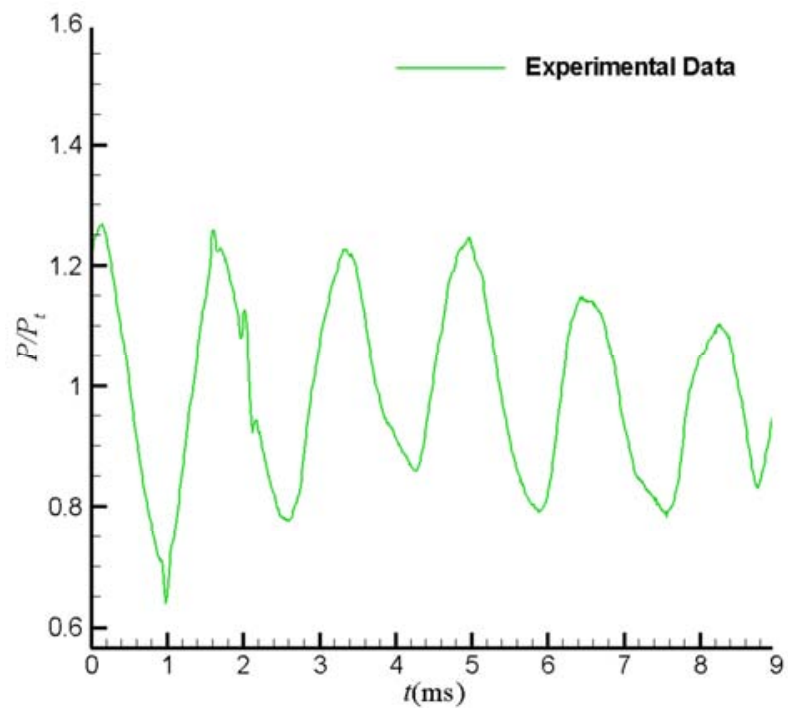

Figure 13. Dynamic pressure signals at cavity base in the FD-14A shock tunnel.

To conclude the results above, for $D / D_{\mathrm{n}} \leq 0.17$, "E1" predicts the shock-standoff distance accurately. For $D / D_{\mathrm{n}} \in$ $(0.17,0.3)$, either "E1" or "E2" is able to predict the shock-standoff distance though the error may exist under some conditions. For the case of $D / D_{\mathrm{n}}=0.5$ and $D / D_{\mathrm{n}}=$ 0.67 , "E3" provides satisfying results of shock-standoff distance. Limited by the amount of experimental and numerical results, the prediction method for shock-standoff distance should be validated further. However, the data which are collected and analyzed in the present paper cover the most of published results of shock-standoff distance for the forward-facing cavity.

The controversy exists on the issue of $L$ in forecasting equation for oscillation frequency. Based on the results above, it is reasonable to believe that the sum of shock-standoff distance and cavity depth should be taken into account to predict oscillation frequency.

\subsection{Oscillation Amplitude and Velocity}

Huebner et al. [6], Sambamurthi et al. [9] and Marquart et al. [10] conducted experimental research on oscillation amplitude at $M_{\infty}=10$. The model is characterized by $D / D_{\mathrm{n}}=0.67$. The normalized amplitude by $D_{\mathrm{n}}$ is plotted in Figure 14. The average values of amplitudes are from $6 \%$ to $12 \%$. It is also noted that the average values of shock velocities are from $2 \%$ to $4 \%$ of $U_{\infty}$ in the upstream and downstream, respectively (Figure 15).

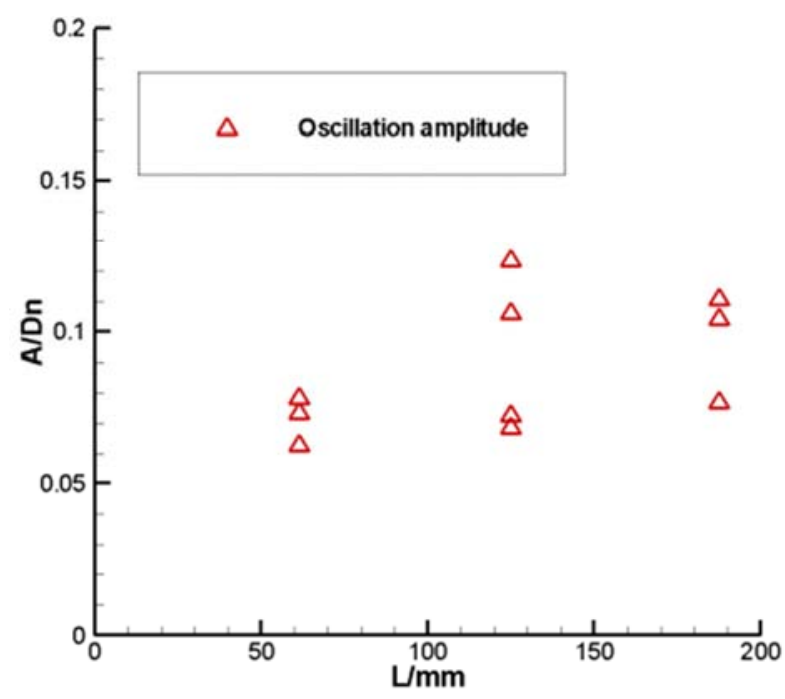

Figure 14. Average of normalized shock amplitude.

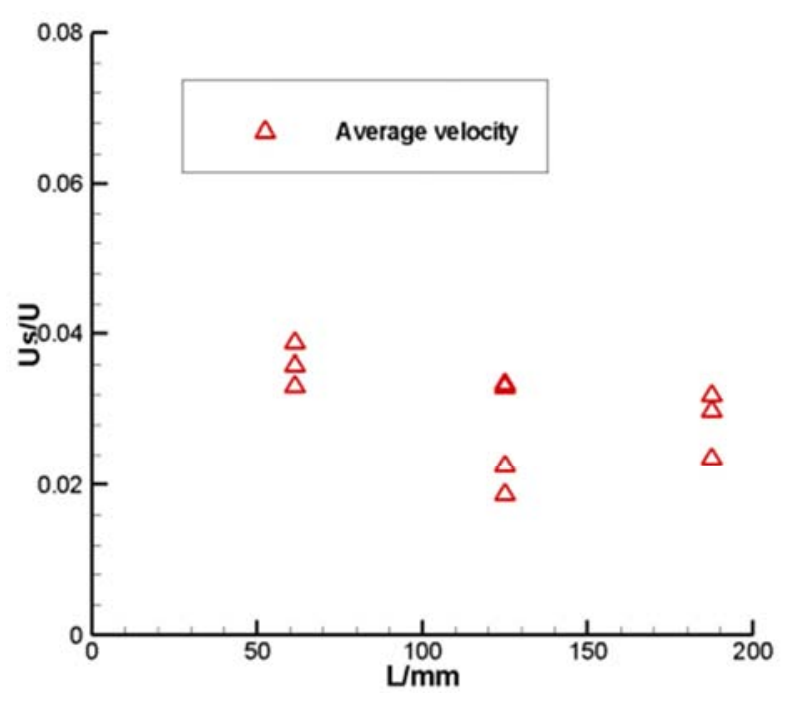

Figure 15. Average of normalized shock velocity.

\subsection{Entropy Distribution}

\subsubsection{Blunt-Nosed Cylinder}

The blunt-nosed cylinders are special conditions of blunt-nosed cones when the cone angle $\theta_{c}$ equals to zero. In this section, the models with small $D / D_{\mathrm{n}}$ are considered which means the Eq. (6) is applied. Constant specific heats $(\gamma=1.4)$ and constant $c_{\mathrm{p}}$ are assumed ahead of and behind 
the shock. The static temperature of flow $T_{\infty}=288 \mathrm{~K}$.

Firstly, from Eq. (19) and Eq. (22), it could tell that the distribution of entropy increase, as well as the entropy gradient, is independent of the nose radius. Secondly, in Figure 16, the entropy increase as the freestream flows across the shock wave is given in detail. Because of the bow shock upstream of the body, the entropy increase is different from one streamline to the next. As the Mach number increases, the profile of non-dimensional $\delta S / \delta S_{\max }$ behaves similar, while the maximum value of the entropy increment rises nearly linearly, as is shown in Figure 17. In Figure 16, the inflexion point of $\delta S / \delta S_{\max }$ represents the extreme position of $d S / d n$. Figure 18 shows that the entropy gradient within the shock layer is negative, and the extreme value varies slightly in the range of $\bar{y} \in(0.8,0.9)$ as the Mach number increases. It should be noted that this intense region of the largest entropy gradients is the main source of the entropy layer, more significant than the nose region. Because the shock waves are stronger and usually highly curved at the higher Mach numbers, the entropy gradients become more severe.

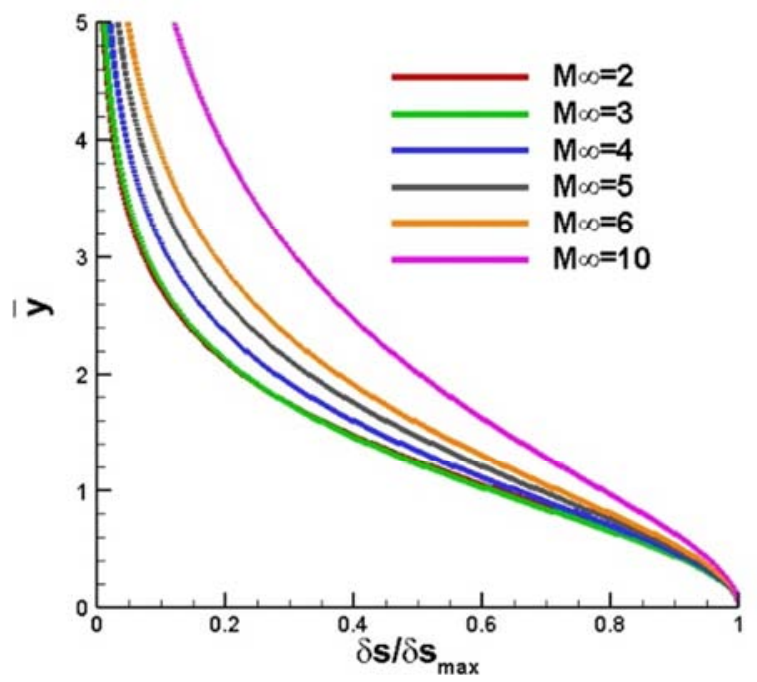

Figure 16. Entropy increase of blunt-nosed cylinders.

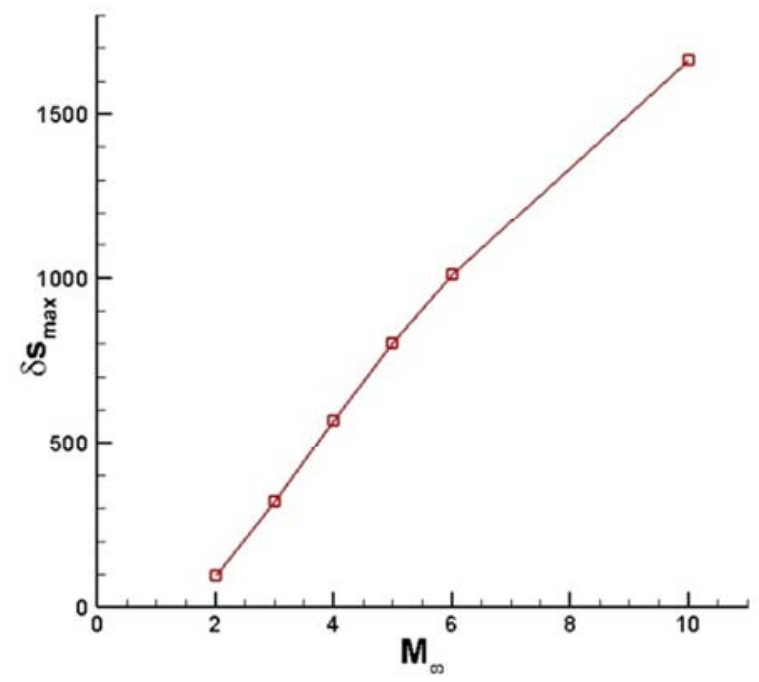

Figure 17. Relation of $\delta S_{\max }$ and $M_{\infty}$ of blunt-nosed cylinders.

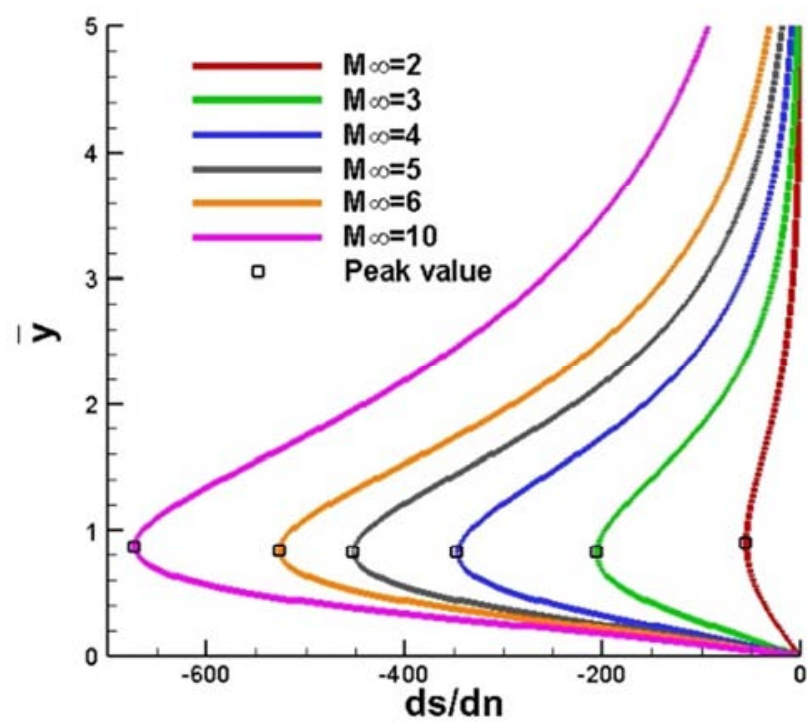

Figure 18. Distribution of $d S / d n$ for blunt-nosed cylinders.

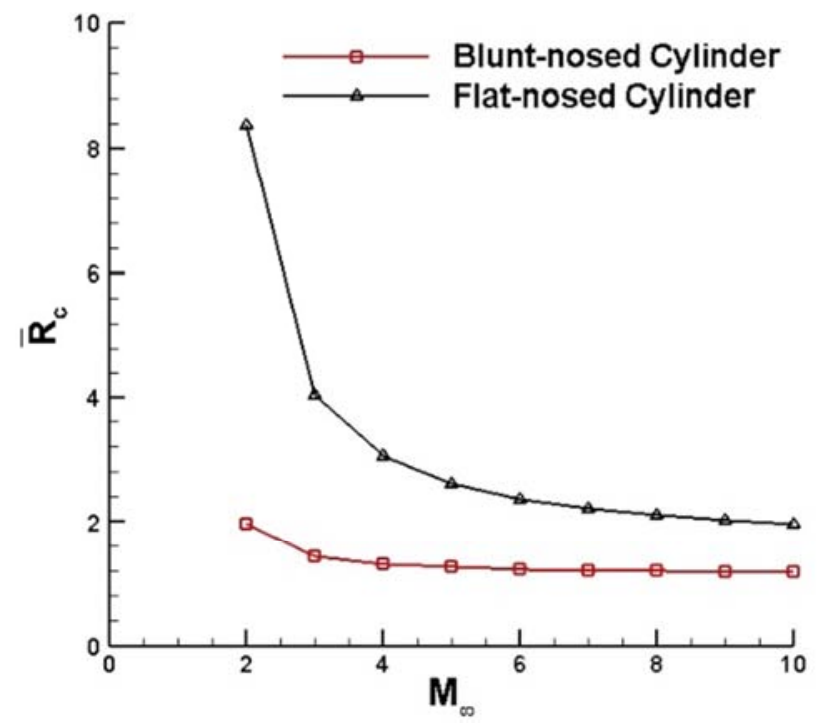

Figure 19. Relation of $M_{\infty}$ and $\overline{R_{c}}$ of blunt-nosed cylinders and flat-nosed cylinders.

\subsubsection{Flat-Nosed Cylinder}

$\overline{R_{\mathrm{c}}}$ represents the curvature of the shock waves to a certain degree. Figure 19 compares the trend of $\overline{R_{\mathrm{c}}}$ with increasing Mach number for blunt-nosed cylinders and flat-nosed cylinders. The $\overline{R_{\mathrm{c}}}$ decreases exponentially as Mach number increases, which suggests the shock wave become even more curved. It can be seen that the shock shape of flat-nose cylinders is much influenced than blunt-nosed cylinders with increasing Mach number. For flat-nosed cylinders, the radius of curvature is about three times larger than blunt-nosed cylinder at the Mach number of 2, then decreases to about two times of the blunt-nosed cylinder at Mach number of 10 .

The distribution of entropy increment for flat-nosed cylinders is given in Figure 20 in non-dimensional form; that is, the $\delta S / \delta S_{\max }$ decreases significantly at the Mach numbers from 2 to 3 , and then almost remain the identical profile as the Mach number increases from 3 to 10 . It is 
suggested that the radius of shock-wave curvature becomes smaller at first, and then follows the Hypersonic Similarity Rule because of the flat nose, even though the Mach number of freestream is just 3 .

The entropy gradient downstream of the shock wave is also investigated. Profiles are illustrated for varied Mach numbers and the slope of entropy gradient is even milder for each Mach number compared with blunt-nosed cylinders, as is shown in Figure 21. What is more, the position of the extreme value moves closer to the coordinate axis of $x$, which leads the position of entropy layer behind the shock wave moves closer to the surface as Mach number increases.

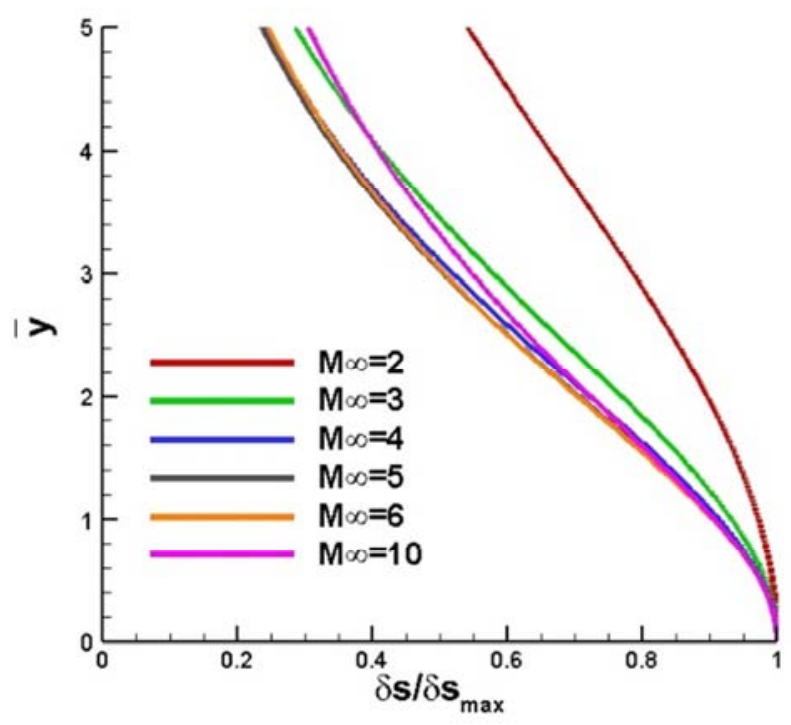

Figure 20. Entropy increase of flat-nose cylinders.

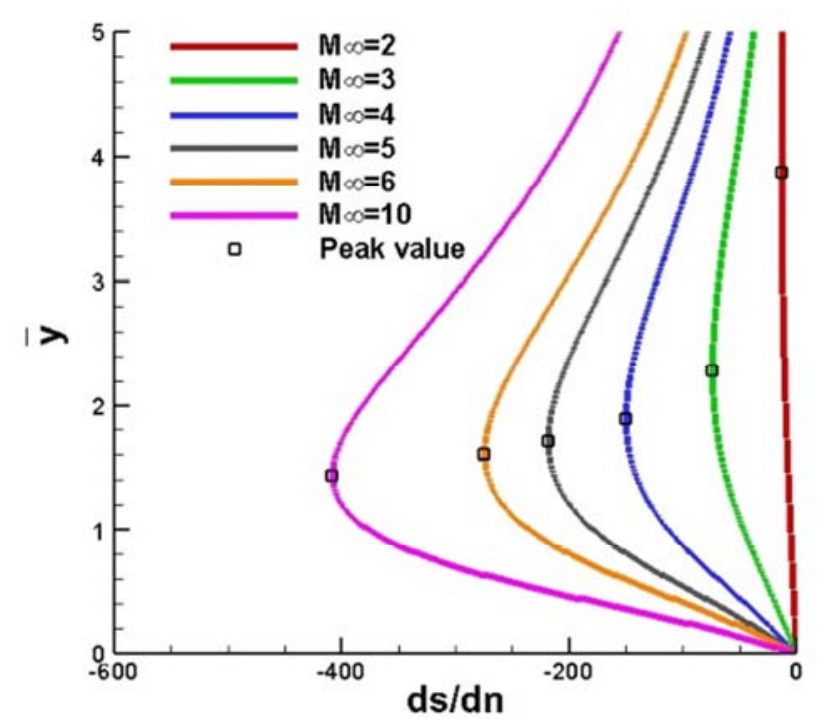

Figure 21. Distribution of $d S / d n$ for flat-nose cylinders.

\section{Conclusion}

Experiments are performed on a flat-nosed cylinder with a forward facing cavity in FD-14A shock tunnel. The predicted results of the shock-standoff distance are approximately equal to the experimental results. A prediction method has been developed for estimating the shock-standoff distance of forward-facing cavity. Giving consideration on varied dimensions of cavities, the prediction method is divided into three cases to predict shock-standoff distance. Then the method is validated based on experimental and numerical results as well as Organ-pipe Theory. What is more, the controversy on the prediction equation of oscillation frequency is discussed.

An analytical method for estimating the distribution of entropy for blunt-nosed cones and flat-nosed cylinders has been developed. The distribution of entropy increase and entropy gradient are both connected with the shape of shock waves directly. As the shock-shape of flat-nosed cylinders is more liable to be influenced than blunt-nosed cylinders with increasing Mach number, the position of the extreme value moves to the surface as the Mach number increases for flat-nosed cylinders, while it remains the identical value for blunt-nosed cylinders.

\section{References}

[1] Engblom, W. A., D. B. Goldstein, and S. P. Schneider, Fluid Dynamics of Hypersonic Forward-Facing Cavity Flow. Journal of Spacecraft and Rockets, 1997. 34 (4): p. 437-444.

[2] Wang, Z., et al., Experimental investigation on drag and heat flux reduction in supersonic/hypersonic flows: A survey. Acta Astronautics, 2016. 129: p. 95-110.

[3] Chou, A., S. P. Schneider, and S. H. Collicott, Measurements of the Interaction of an Upstream Laser Perturbation with a Forward-Facing Cavity, in 51st AIAA Aerospace Sciences Meeting including the New Horizons Forum and Aerospace Exposition2013: Grapevine, Texas.

[4] Yadav, R. and U. Guven, Aerothermodynamics of a hypersonic vehicles with a forward facing parabolic cavity at nose. J Aerospace Engineering, 2014. 228 (10): p. 1863-1874.

[5] Yadav, R. and U. Guven, Aerodynamic Heating of a Hypersonic Projectile with Forward-Facing ellipsoid Cavity at nose. Journal of Spacecraft and Rockets, 2015. 52 (1): p. 157-165.

[6] Huebner, L. D. and L. R. Utreja, Mach 10 Bow-shock Behavior of a Forward-Facing Nose Cavity. Journal of Spacecraft and Rockets, 1993. 30 (3): p. 291-297.

[7] Hartmann, J. and B. Troll, On a New Method for the Generation of Sound Waves. Phys. Rev., 1922. 20: p. 719-727.

[8] Ladoon, D. W., S. P. Schneider, and J. D. Schmisseur, Physics of Resonance in a Supersonic Forward-Facing Cavity. Journal of Spacecraft and Rockets, 1998. 35 (5): p. 626-632.

[9] Sambamurthi, J. K., L. D. Huebner, and L. R. Utreja, Hypersonic Flow Over a Cone With Nose Cavity, in AIAA 19th Fluid Dynamics Plasma Dynamics and Lasers Conference1987: Hawaii.

[10] Marquart, E. J. and J. P. Grubb, Bow Shock Dynamics of a Forward-facing Nose Cavity, in AIAA 11th Aeroacoustics Conference 1987: Sunnyvale. 
[11] Engblom, W. A., et al., Fluid dynamics of hypersonic forward-facing cavity flow. AIAA Journal, 1996. AIAA 96-0667.

[12] Engblom, W. and D. Goldstein, Acoustic Analogy for Oscillations Induced by Supersonic Flow over a Forward-Facing Nose Cavity, in 47th AIAA Aerospace Sciences Meeting Including the New Horizons Forum and Aerospace Exposition2009: Orlando, Florida.

[13] Yuceil, K. B. and D. S. Dolling, Nose Cavity Effects on Blunt Body Pressure and Temperature at Mach 5. Journal of Thermophysics and Heat Transfer, 1995.9 (4): p. 612-619.

[14] Billig, F. S., Shockwave shapes around spherical and cylindrical nosed bodies. Journal of Spacecraft and Rockets, 1967. 4 (6): p. 822-823.

[15] Engblom, W., et al., Hypersonic Forward-facing cavity flow: an experimental and numerical study, in 33rd Aerospace Sciences Meeting and Exhibit1995: Reno.
[16] Engblom, W. A., et al., Experimental and Numerical Study of Hypersonic Forward-Facing Cavity Flow. Journal of Spacecraft and Rockets, 1996. 33 (3): p. 353-359.

[17] Yuceil, K. B. and D. S. Dolling, IR imaging and shock visualization of flow over a blunt body with a nose cavity, in AIAA 34th Aerospace Sciences Meeting and Exhibit1996: Reno.

[18] Saravanan, S., G. Jagadeesh, and K. P. J. Reddy, Investigation of Missile-Shaped Body with Forward-Facing Cavity at Mach 8. Journal of Spacecraft and Rockets, 2009. 46 (3): p. $577-591$.

[19] Juliano, T. J., et al., Starting Issues and Forward-Facing Cavity Resonance in a Hypersonic Quite Tunnel, in 38th Fluid Dynamics Conference and Exhibit2008: Seattle, Washington.

[20] Segura, R., Oscillations in a Forward-Facing Cavity Measured Using Laser-Differential Interferometry in a Hypersonic Quiet Tunnel, 2007, Purdue University: West Lafayette, Indiana. 